Rather intriguingly, these relatively simple mechanical measurements serve to specify the first three orders of the electrical characteristics of the double layer. If the dipole moment per unit area of the double layer is $\mathrm{k}^{\prime}$, the total dipole moment of the surface is $\mathrm{k}^{\prime} \mathrm{A}$, and is directed along the $\mathrm{X}$ axis. Since the intersection of the principal axes of inertia coincides with the center of gravity, all first-order (static) moments of the lamina are zero; analogously, all five quadripolar components of the double layer vanish in the frame of reference which we have selected. Five of seven possible octapolar components also vanish, leaving only $a_{30}=-\left(3 k^{\prime} / 2 k\right)\left(I_{y}+I_{z}\right)$, and $a_{32}=\left(k^{\prime} / 4 k\right)\left(I_{z}-I_{y}\right)$. Since the $a_{32}$ component reflects the difference between the maximal and minimal moments of inertia of the lamina, it vanishes in the case of a circular rim or, more generally, in the case of a rim with tetragonal symmetry about the $\mathrm{X}$ axis.

The preceding hypothetical example is relatively simple, not only because the rim of the double layer is planar, but also because a "local" reference system was adopted in which all fifteen components of the first three orders vanish save for one dipolar and two octapolar components. This simplification is permissible, but the description is not complete unless the location and orientation of the local system within the usual anatomic reference coordinates is specified.

The mathematical complexities of the situation increase considerably when the rim of the double layer is nonplanar and/or the components are referred to the anatomic rather than the local coordinate system. These complexities tend to obscure the physical interpretation of double-layer activity as mechanical analogues, but they do not appear to introduce excessive computational difficulties.

The recent electrocardiographic literature contains two closely related proposals for determining the equivalent generator components of the heart from body-surface measurements of potential..$^{5,6}$ If the body, including the entire cardiac region, were an electrically homogeneous, linear, and isotropic volume conductor, the equivalent generator components determined from body-surface measurements of potential would be identical to the intrinsic electrocardiographic properties obtained by the type of direct analysis of double layers outlined above. Since it is most unlikely that the body meets these highly idealized conditions, we would anticipate differences between the equivalent and intrinsic multipolar components of the cardiac generator, and these differences should to some extent reflect distortion produced by the actual volumeconductor properties of the body as compared to the hypothetically ideal situation. It is conceivable that animal experiments can be designed for the purpose of testing in this way the electrocardiographic distortions produced by the passive electrical properties of a given subject.

\section{Daniel A. Brody, M.D. Department of Medicine University of Tennessee Memphis, Tennessee}

\section{REFERENCES}

1. Frank, E.: A comparative analysis of the eccentric double-layer representation of the human heart, AM. HEART J. 46:364, 1953.

2. Brody, D. A.: Limited reliability of precordial electrodes as vectorcardiographic leads, Science $125: 352,1957$.

3. Yeh, G. C. K., and Martinek, J.: Multipole representation of an eccentric dipole and an eccentric double layer, Bull. Math. Biophysics $21: 33,1959$.

4. Brody, D. A., and Bradshaw, J. C.: The equivalent generator components of uniform double layers, Bull. Math. Biophysics (in press).

5. Geselowitz, D. B.: Multipole representation for an equivalent cardiac generator, Proc. Inst. Radio Engineers 48:75, 1960.

6. Brody, D. A., Bradshaw, J. C., and Evans, J. W.: A theoretical basis for determining heart-lead relationships of the equivalent cardiac multipole, I.R.E. Trans. on Bio-Medical Electronics 8:139, 1961.

\title{
Potassium depletion and benzothiadiazine drugs: A source of overconcern?
}

The diuretic and antihypertensive properties of a variety of benzothiadiazine compounds have been exploited widely in the treatment of edematous states, such as congestive heart failure, discases $\mathrm{cm}$ bracing the nephrotic syndrome, or cirrhosis of the liver, and in the treatment of hypertensive disease. When these natriuretic agents were first introduced, it was recognized that they lowered the level of serum potassium in some patients and also elevated the plasma carbon-dioxide combining power. ${ }^{1}$ An early report dealt with the expected occurrence of digitalis toxicity, apparently the result of chlorothiazide causing loss of potassium. ${ }^{2}$ With the development and introduction of each subsequent modification of the benzothiadiazine structure came an assessment of the extent of the urinary excretion of potassium that it would cause. The major advantage stressed for these newer thiazides, other than increased potency, has been the more "favorable" ratio of sodium to potassium that they produce in the urine. Supplementation of the intake of potassium of patients treated with thiazide drugs, either by adding foods 
high in potassium content to the diet, or by giving oral potassium salts, has been advocated and frequently utilized. Combinations of the thiazides and potassium salts have been made available commercially to fill this "need" for potassium supplementation.

Is all this concern about potassium depletion valid? It is well known that the concentration of an electrolyte in the serum need not correlate with its total content in the body. The presence of hypokalemia cannot be taken as indubitable evidence of cellular potassium deficiency. Potassium depletion can be accompanied by hypokalemia and also by an extracellular alkalosis, with elevation of the plasma carbon-dioxide combining power, since hydrogen ions enter cells when the cells lose potassium ions. However, the level of serum potassium may also fall without depletion of the total body potassium, as a simple consequence of a metabolic alkalosis which causes the migration of potassium ions into cells. A low serum potassium and a high carbon-dioxide combining power are common to both of these situations. In the case of thiazide administration, which is the primary element? Is it the loss of potassium from the body, or is it the institution of a metabolic alkalosis? This question requires further investigation.

The evaluation of the magnitude of the urinary loss of potassium which results from the use of benzothiadiazine drugs usually has been carried out over a period of time limited to a few days. It is true that the initiation of thiazide therapy does increase the urinary excretion of potassium. This has been stressed so much that the scattered voices which offer evidence that the chronic administration of these agents need not lead to potassium deficiency have been virtually overlooked. Talso and Carballo ${ }^{3}$ studied 71 patients who were given hydrochlorothiazide or benzydroflumethiazide daily for 28 days. Twenty of these individuals also received supplementary potassium salts. The mean total exchangeable body potassium, determined prior to and at the end of this course of treatment, irrespective of the addition or omission of potassium supplementation, showed no significant change. Gifford and associates, ${ }^{4}$ although finding a slight reduction in exchangeable body potassium in the first 2 weeks of treatment, ohserved that the mean value for 5 patients in subsequent weeks actually increased. Likewise, experimental animals show little evidence of potassium deficiency after the prolonged administration of thiazides. Delahunt ${ }^{5}$ has given large amounts of polythiazide $(450 \mathrm{mg}$. per kilogram of body weight per day) to dogs for 6 months without altering the potassium content of their skeletal muscle. Sones and associates ${ }^{6}$ gave chlorothiazide to animals for 5 months without producing a change in total body exchangeable potassium.

It appears that the renal tubules of most patients and experimental animals have the ability to counteract the loss of potassium which may occur in the initial phase of thiazide administration, and can correct any resulting negative balance of potassium in spite of continued daily use of these drugs. The weight of evidence to date supports the view that the hypokalemia which may be present with prolonged thiazide treatment represents a deviation from the normal intracellular-extracellular potassium concentration gradient and not a depletion of the body's stores of potassium. It is, therefore, a relatively benign state, except for its possible potentiation of digitalis toxicity. The concern about potassium depletion with prolonged administration of benzothiadiazine drugs is unjustified, and the deliberate routine supplementation of the intake of potassium over long periods of time should be abandoned.

John M. Weller, M.D. University of Michigan Medical School Ann Arbor, Mich.

\section{REFERENCES}

1. Weller, J. M., Reynolds, E. W., and Judge, R. D.: Clinical evaluation of the diuretic drug chlorothiazide, Univ. of Mich. Med. Bull. 24:44, 1958.

2. Schreiner, G. E., and Bloomer, H. A.: Effect of chlorothiazide on the edema of cirrhosis, nephrosis, congestive heart failure, and chronic renal insufficiency, New England J. Med. 257:1016, 1957.

3. Talso, P. J., and Carballo, A. J.: Effects of benzothiadiazines on serum and total body electrolytes, Ann. New York Acad. Sc. 88:822, 1960.

4. Gifford, R. F., Jr., Mattox, V. R., Orvis, A. L., Sones, D. A., and Rosevear, J. W.: Effect of thiazide diuretics on plasma volume, body electrolytes, and excretion of aldosterone in hypertension, Circulation 24:1197, 1961.

5. Delahunt, C. S.: Personal communication.

6. Sones, D. A., Wakim, K. G., Orvis, A. L., and McGuckin, W. F.: Effects of chlorothiazide on body electrolytes, Fed. Proc. 19:249, 1960. 